\title{
Measurement of Some Antioxidants in Serum of Infected Pregnant Women with Toxoplasmosis
}

\author{
Muna I. Ibraheem \\ Office of \\ Agricultural
}

Layla A. Mustaf

Nabeel E. Salih

Department of Chemistry

Department of Biology

Research/ Ninevah

College of Science

College of Education for Pure

Science

Mosul University

Mosul University

Munaismai112@yahoo.com laylamustaf@gmail.com

nabeelsalih@gmail.com

DOI: $\underline{10.33899 / \text { edusj.1970.163329 }}$

Received

$21 / 11 / 2018$
Accepted

27 / 02 / 2019

\begin{abstract}
The study involved measurement of medical, biological and antioxidant importance of toxoplasmosis and the effect of the causative agent, Toxoplasma gondii ,on the level of the biochemical parameters in the serum of 225 pregnant women infected with toxoplasmosis compared with225serum of women infected as control group. The criteria taken into consideration are: Glutathione (GSH), Albumine (Alb) , Uric acid (UA), Malondialdehyde (MDA), Peroxinitrate (No).The Results showed: A significant increase $(p \leq 0.01)$ in level of GSH, MDA and NO whereas Alb and U.A decreased, compared to control group. Interference between infection and pregnancy periods showed an significant increase $(\mathrm{p} \leq 0.01)$ in level of GSH during 1-3 months pregnancy period and MDA and non- significant increase in level of NO during 6-9 months pregnancy period. Alb and UA levels decreased, as significantly $(p \leq 0.01)$ in serum of pregnant infected women.
\end{abstract}

Keywords: Toxoplasmosis , Antioxidant, Malondialdehyde , Peroxinitrate. 


\section{قياس بعض مضادات الأكسدة في مصل النساء الحوامل المصابة بداء المقوسات الكونديه}

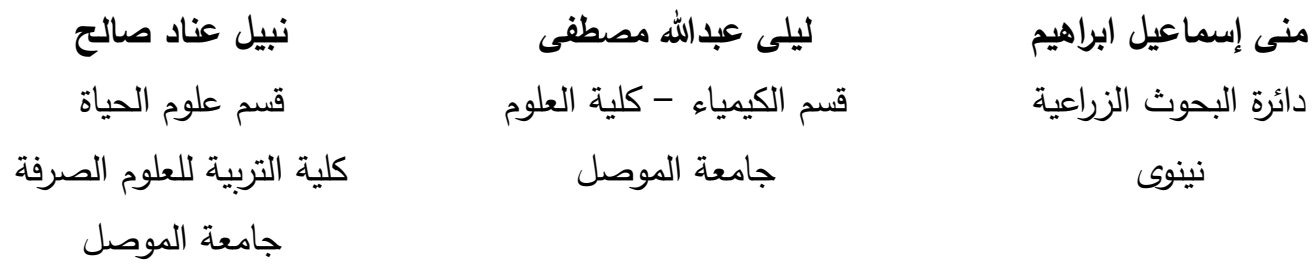

\begin{tabular}{|c|c|}
\hline القبول & الاستلام \\
\hline 019 /02 / 27 & 2018 / 11 / 21 \\
\hline
\end{tabular}

nabeelsalih@gmail.com

laylamustaf@gmail.com

Munaismail12@yahoo.com

DOI: $\underline{10.33899 / \text { edusj.1970.163329 }}$

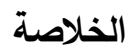

تناولت الدراسه جوانب مهمة من النواحي الطبية، البيولوجية والكيموحيوية لداء المقوسات Toxoplasmosis الأكسدة في مصل 225 من النساء الحوامل المصابة بداء المقوسات مقارنة بمصل 225 من النساء غير

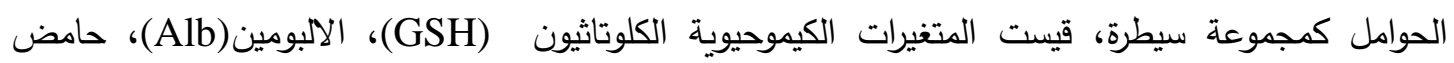

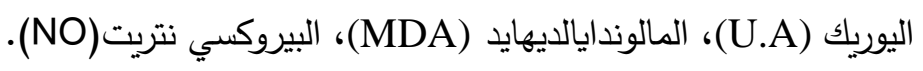



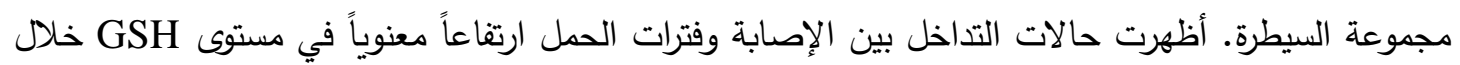
فترة الحمل (1-3) أشهر ، وارتفاعاً معنويا في مستوى MDA وغير معنوي في مستوى NO خلاد لإل فترة الحمل

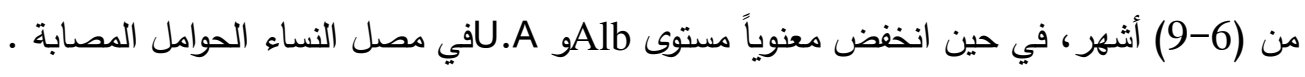
الكلمات المفتاحية: داء القطط, مضادات اكسده ركلوناثايون , مالوندالديهايد , بيروكسي نتريت .

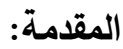

تعد المقوسة الكوندية من الاوالي الطفيلية الداخل خلوية إجباريا، وتعد القطط وجميع الأنواع التابعة

للعائلة السنورية مضائف نهائية إجبارية للطفيل ومصدر الإصابة الرئيسي[11]. في حين أن عددا كبيرا من الزواحف والطيور واللبائن، بضمنها الإنسان، تعد مضائف وسطية [2]. وتمتلك الأطوار المختلفة للطفيل

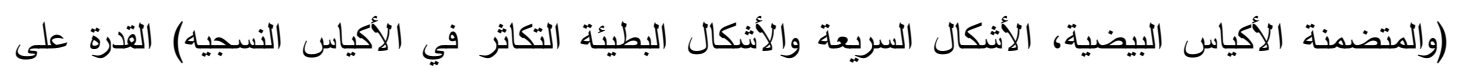


اصابة المضائف الوسطية والنهائية. كما يمكن أن ينتقل الطفيل من الأم إلى الجنين عن طريق المشيمة

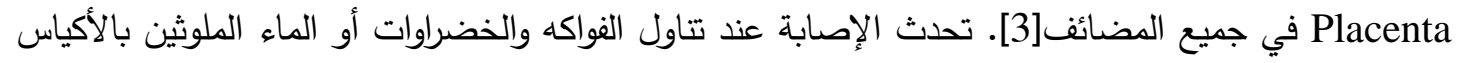
البيضية المتبوغة أو التماس مع التربة الملوثة، أو بصورة غير مباشرة عند تتاول اللحوم الحاوية على الأكياس

النسيجية غير المطهية جيدا أو قد تحدث الإصابة بانتقالها عن طريق المشيمة من الأم إلى الجنين [4].

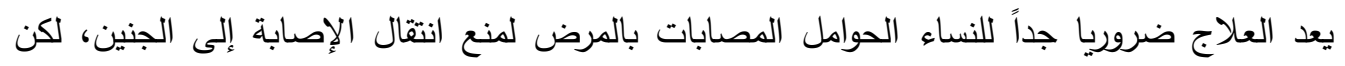



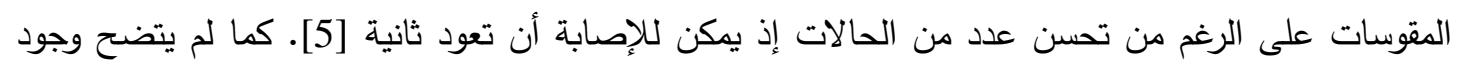

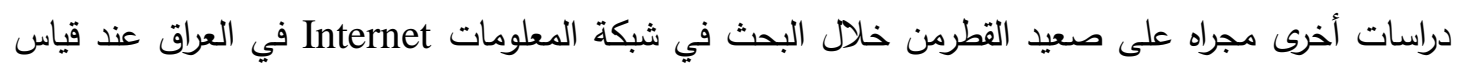
المتغيرات الكيموحيويه المتتاوله في البحث وكان الهدف من البحث دراسة تأثير داء المقوسات على مستوى



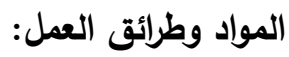
جمع عينات المشيمة:

جمعت عينات المشيمة من نساء مجهضات وغير مجهضات وبعدد 24 عينة من مستشفى الخنساء

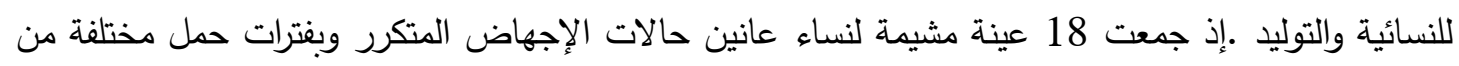


(Normal Saline

المباشر لمستخلص المشيمة.

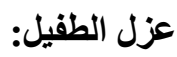

تم عزل الطفيل بصورته النقية من المشائم المخدجة [6]؛[7]؛[8].

مجموعة النساء المصابات وغير المصابات بداء المقوسات الكوندية

جمعت 450 عينة دم لنساء حوامل غير مصابات كمجموعه سيطرة ومصابات بداء المقوسات

الكوندية. شخصت حالتهن من قبل طبيبات مختصات في مستثفى الخنساء للنسائية والتوليد /الثعبة

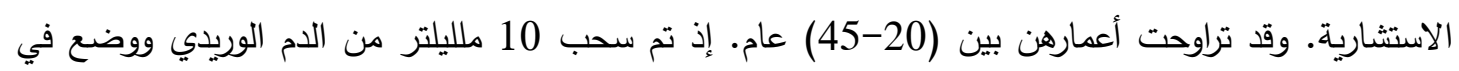

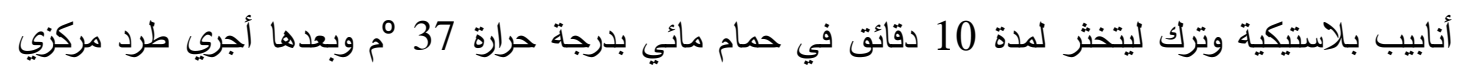

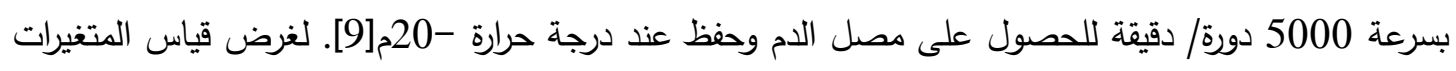
الكيموحيوية. أجريت عدة قياسات كيموحيوية في دم النساء الحوامل المصابات وغير المصابات بداء المقوسات دورات

تم تقدير الكلوتاثيون في مصل الدم باستخدام الطريقة المحورة المتبعة لدى الباحث [9]. 
استخدمت فيها عدة محاليل جاهزة من شركة SYRBio والتي تعتمد على كمية الألبومين الذي يربط

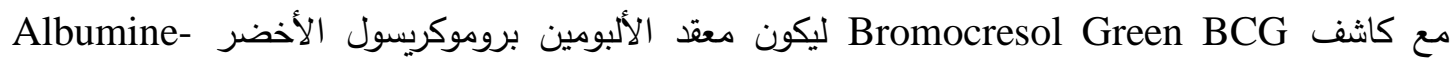

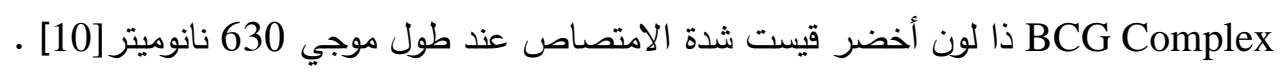
تقدير حامض اليوريك:

استخدمت طريقة إنزيمية في تقدير حامض اليوريك في المصل باستخدام محاليل جاهزة من شركة

.[9] SYRBio

تقدير مستوى بيروكسدة الدهن(المالوندايالديهايد):

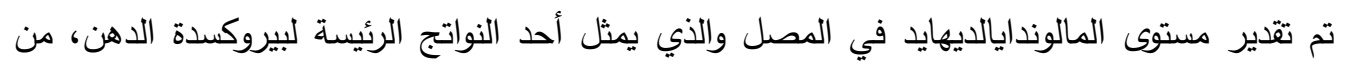
خلال التفاعل بين بيروكسيدات الدهن وبشكل رئيس المالوندايالديهايد وبين حامض ثايوباربيوتاريك (TBA) .[11] Thiobarbituric acid تقدير مستوى البيروكسي نتريت قدر جذر البيروكسي نتريت باستخدام الطريقة المحورة للباحثين [12].

\section{النتائج والمناقشة:}

نتائج قياس مستوى المتغيرات الكيموحيوية في مصل النساء الحوامل المصابة وغير المصابة اظهرت النتائج الموضحة في الجدول(1) إلى وجود تأثير معنوي عالٍ في مستوى كل من الكلوتاثيون ،

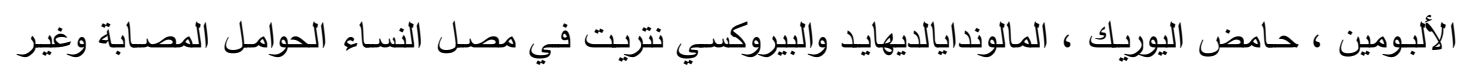
P المصابة وكذلك خلال فترات الحمل والتداخل بين حالات الإصابة وفترات الحمل عند مستوى احتمال

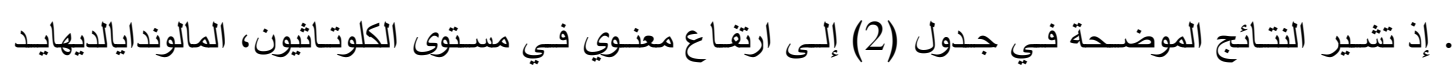

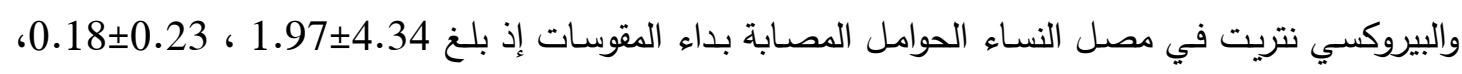

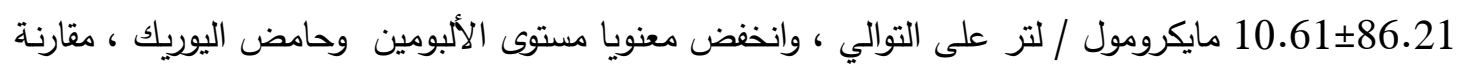

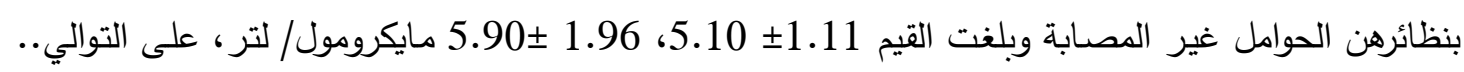
وقد يرجع السبب إلى كثرة استخدام مضادات الأكسدة مثل الفيتامينات ولاسيما فيتامين E الذي يساعد في ارتفاع نسبة الكلوتاثيون نسبياً في مصل الدم للأشخاص المصابين بالمرض حيث إن فيتامين Eيساعد في عمليات

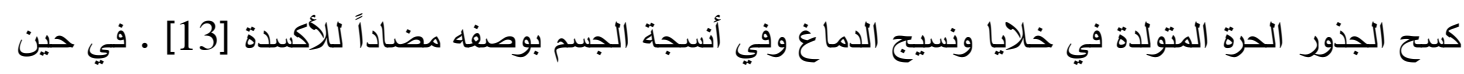
ارتفاع المالوندايالديهايد والبيروكسي نتريت نتج عن الكرب التأكسدي في حالات الحمل وكافة الحالات المرضية





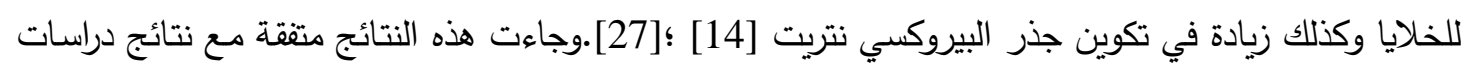
لأمراض طفيلية أخرى ومرضى سرطان الرئة[15] ؛ [16] ؛ ؛ 
الجدول1 : يوضح المعدل والانحراف القياسي للمتغيرات الكيموحيوية في مصل النساء الحوامل المصابة وغير

المصابة.

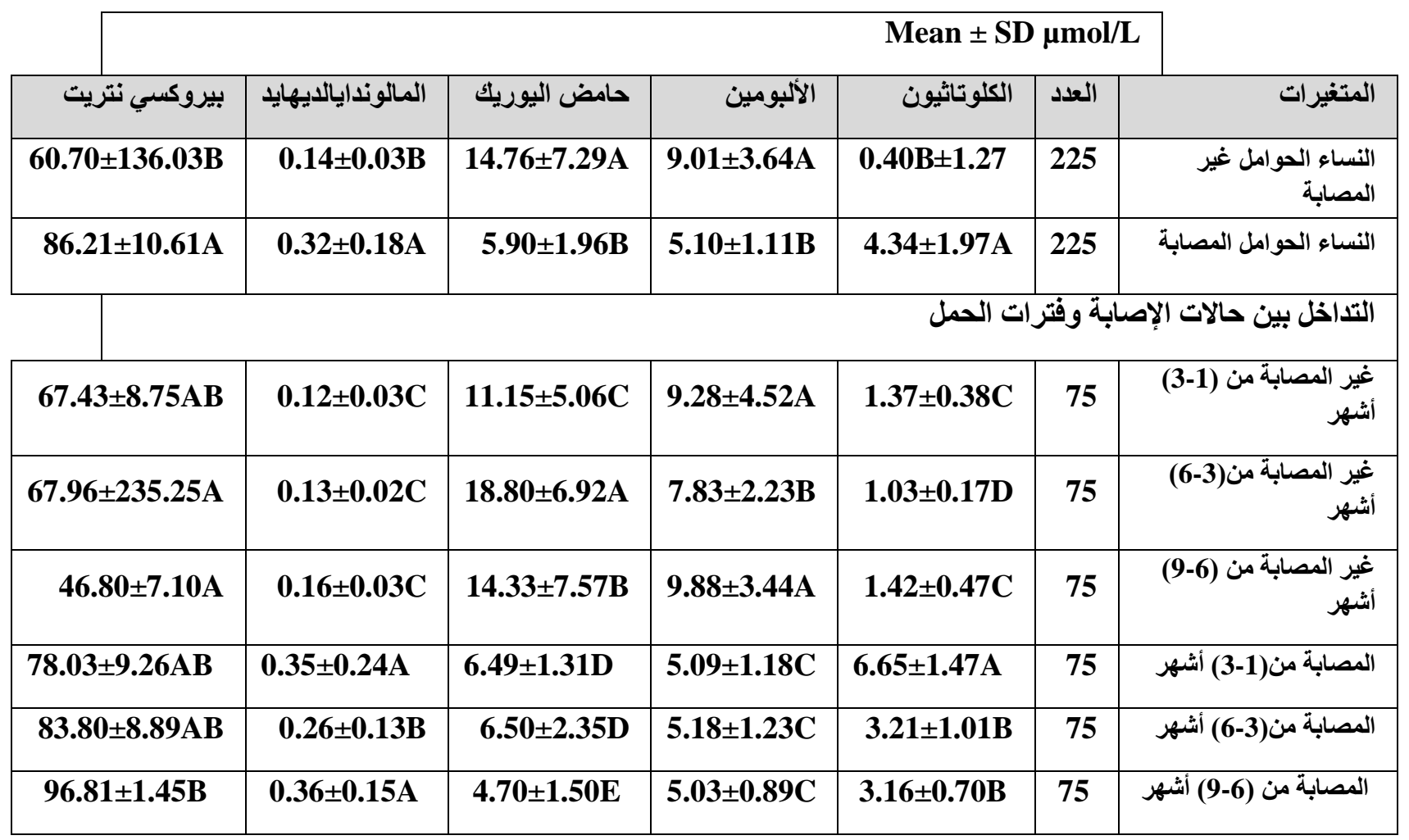

Within columns, means having the same letters do not differ significantly at $\mathbf{p} \leq$ 0.05 probability level.

مستوى المتفيرات الكيموحيوية في مشائم النساء الحوامل المصابة وغير المصابة

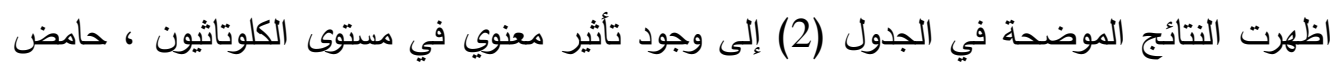

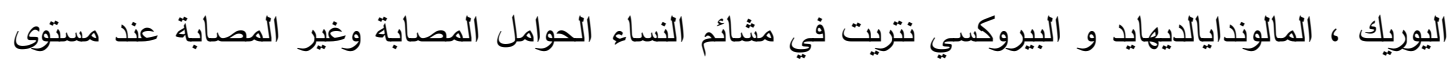

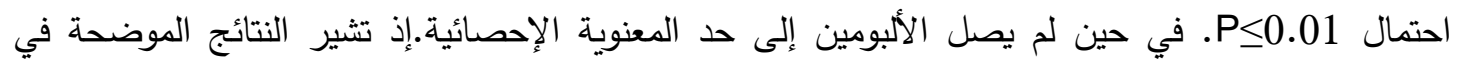
الجدول(2) إلى وجود ارتفاع معنوي في الكلوتاثيون وحامض اليوريك في مشائم النساء الحوامل المصابة خلال الدين فترة الحمل 9-6أشهر وبلغ 2.32×06.06، 4.16.14.11مايكرومول / لتر على التوالي ، وارتفع معنويا

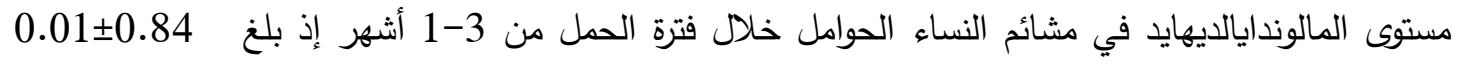

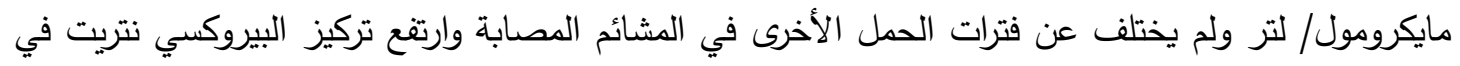





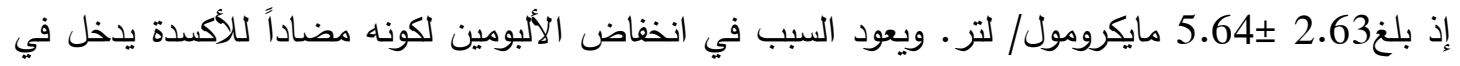

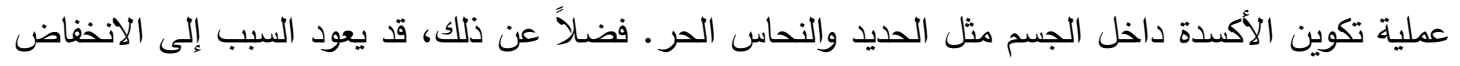

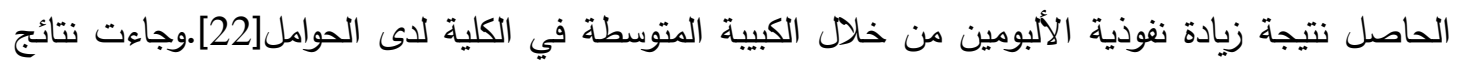



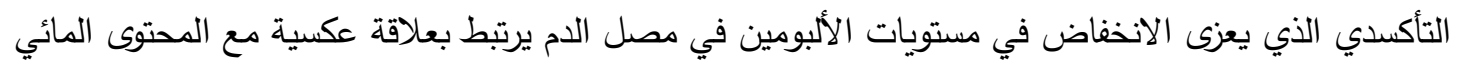
لاى النساء الحوامل ويعد عامل تخفيف الذي يكون مرتبطاً مع توسع في حجم البلازما خلال الحمل هو العامل 
الرئيسي الذي يسبب انخفاضاً في البومين المصل[23] . . ومرضى داء السكر ومرضى سرطان المعدة وأورام الخلايا الظهارية للفم، سرطان الرئة [24]؛ [25]؛ [26]؛ [27] وقد يعود السبب في ذلك إلى النقص الغذائي أو لدوره في حجب الجذور الحرة من خلال الارتباط بالأحماض الدهنية الحرة [ 28] ومركبات الهايبوكلورايد Hypochloride (HOCL



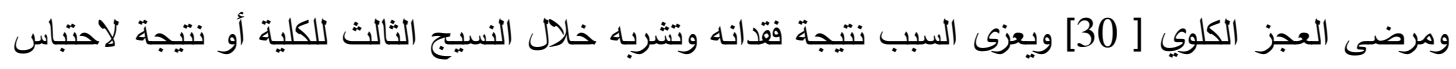
السوائل.

جدول 2: يوضح المعل والانحراف القياسي للمتغيرات الكيموحيوية في مشائم النساء الحوامل المصابة وغير

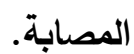

\begin{tabular}{|c|c|c|c|c|c|c|}
\hline \multicolumn{5}{|c|}{ Mean \pm SD $\mu \mathrm{mol} / \mathrm{L}$} & \multirow[b]{2}{*}{  } & \multirow[b]{2}{*}{ الحمل المائم خلال فترة } \\
\hline البيروكسي نتريت & المالوندايالديهايد & حامض اليوريك & الألبومين & الكلوتاثيون & & \\
\hline 87.72 $\pm 23.69 A B$ & $0.28 \pm 0.15 B$ & $10.08 \pm 3.87 \mathrm{BC}$ & $9.25 \pm 5.69 \mathrm{~A}$ & $1.17 \pm 0.47 B$ & 6 & مشائم غير المصابة \\
\hline $75.5 \pm 0.01 B$ & $0.84 \pm 0.01 \mathrm{~A}$ & $6.68 \pm 0.01 C$ & $6.42 \pm 0.01 \mathrm{~A}$ & $1.47 \pm 0.01 B$ & 6 & مصابة من (3-1) أشهر \\
\hline $104.31 \pm 0.01 A$ & $0.80 \pm 0.01 \mathrm{~A}$ & $10.91 \pm 0.03 \mathrm{AB}$ & $5.64 \pm 2.63 \mathrm{~A}$ & $1.44 \pm 0.04 B$ & 6 & مصابة من (3-6) أشهر \\
\hline $93.40 \pm 22.92 \mathrm{AB}$ & $0.74 \pm 0.12 \mathrm{~A}$ & $14.11 \pm 4.16 \mathrm{~A}$ & $8.28 \pm 0.14 \mathrm{~A}$ & $2.32 \pm 0.06 \mathrm{~A}$ & 6 & مصابة من (6-9) أشهر \\
\hline
\end{tabular}

Within columns, means having the same letters don't differ significantly at $\mathbf{p} \leq$ 0.05 probability level

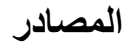

1- Remington JS and Demonts G (1976) Toxoplasmosis. In Remington JS; Kelein JD (Eds) Infections Diseases of the Fetus and New Born Infants, ( $1^{\text {st }}$ ed.)W.B. saunders Co.,Philadelphia.

2- Tenter AM; Heckeroth AR and Weiss LM (2000). Int. J.Parasitol.,30:1217-1258.

3- Roberts LS and Janovy J (1996) Foundation of Parasitology . ( $5^{\text {th }}$ ed.). London, C. Brown, 121-135.

4- Roberts LS and Janvoy J (2000) Foundation of Parasitology. (6th ed.).Mc GrawHill Companies, London, 127-132.

5- Wieffer M;Gobbels M Jand Boris S (2005) Molecular and Biochemical Parasitology. Center for tropical and emerging global diseases, University of Gearyia,724. Biological Sciences Building, Athens, GA 30602,USA,137(1):99110.

6- Sharma SP and Dubey JP(1981) Quantitative survival Toxoplasma gondii tachyzoites and bradyzoites in pepsin and trypsin solution.Ann. J.Vet. Res.,42:128130. 
7- Dubey JP and Livington JR (1986) Sarrcocystis capracanis and Toxoplasma gondii infection in range goat from Texas. Am. J.Vet.,47(3):523-524.

8- AL Khaffaf, F.H.O.(2001) Isolation and study of epidemiological seroprevalence of toxoplasmosis age in Nineveh province. M.Sc. Thesis .College of Sciences, University of Mosul. Iraq.

9- Tietz NW (1999) Textbook of Clinical Chemistry. ( $3^{\text {rd }}$ edn.) W.B.Saunders Company, Philadephia,1239-1250.

10- Doumas BT; Briggs HG (1972) Standard Method of Clinical ChemistryAcad.Press. N.Y.,7:175-188.

11- Wysocka RW; Wysocki H; Buks H; Zozulinskay D; Wykretowic ZA and Kazmierczak M (1995) Metabolic control quality and free redical activity in diabetic patients. Diab . Res. Clin. Prac.,27:193-197.

12- Vanuffelen BE; Van Derzo J and Dekoster BM (1998) Biochem. J.330-719.Cited by Al - Zamely (2001).

13- Zheng X (2003) Tocopheral, Lipidantioxidant department of fire radical and radiation biology. The University of Iowa City. JA.52242-1181.

14-Gupta SS;Malhotra N; Sharma D; Chandra A and Askok A (2009) Oxidative stress and its role in female infertility and assisted reproduction.Clin.Impl.,2(4):147-167.

15- Kiral F; Karagenc T; Pasa S; Yenisey C and Seyrek K (2005) Dogs with Hepatozoon canis respond to the oxidative stress by increased production of glutathione and nitric oxide . Vet. Parasitology. Vol.,131(2):15-21.

16- Kaynar H; Meral H; Turhan H; Keles M; Celik G and Acay F (2005) Glutathione peroxidase, glutathione-S- Tansferase, catalase, xanthine oxidase $\mathrm{Cu}-\mathrm{Zn}$ superoxide, dismutase activities, total glutathione, nitric oxide, and malondialdehyde levels in erythrocytes of patients with small cell and non cancer Lett.28,227(2):133-139.

17- Romao PR; Tovar J; Fonseca SG; Moraes RH; Gruz AK; Hothersall JS; Noronha- Dutra AA; Ferreira SH and Cunha FQ (2006) Glutathione and theredox control system trypanothione/ trypanothione reductase are involved in the protection of Leishmania spp.Against nitrosothial- induced cytotooxicity Braz. J. Med.Biol.Res.,39(3):355-363.

18- EL- Badry AA(2006). J. T.V. Med. Sci.,1(1):57-64.

19- Crnoga M; Petievski R; Mrlyak V; Kis I; Torti M; Kucer N; Matijatko V;Sacer I and Stokovic I (2010). Vet. Med.55(4):163-171.

20- Fidan AF; Cingi CC; Kara fakioglu YS; Vtuk AE; Pekaya S and Piskin FC (2010). J. Animal Vet., 9(12):1707-1711. 
21- Idonije OB; Festus O; Okhiai O; Akpamu V (2011). Asian. J. Biol. Sci.,4:506513.

22- Iqpal SA; Khan MA; Khan MI and Fayaz KM (2004). The PRO.11(3):278-284.

23- Khetsuriani T; Sanikidze T and Khugashvili R (2004). Ann.Bio. Med. Res. Edu. Cat.,4(1):34-36.

24-AL-Hadidi,A,A,M (2005). M.Sc. Thesis in Biochemistry ,College of Sciences, University of Mosul. iraq.

25-AL-guresiy,A.T.Y (2005). M.Sc. Thesis in chemistry ,College of Sciences, University of Mosul.Iraq.

26- Dawood,R,M(2008).Ph.D. Thesis. University of Baghdad.Iraq.

27- Mla-ALw .F. Y.H (2011). M .Sc. Thesis in Biochemistry. College of Sciences, University of Mosul.Iraq.

28- Robert R; Jarrige PL; Mahaza C; Cottin J; Marot A and Senet J (1991). Inf. Immn.,4670-4673.

29- AL-Sabaawy , O,M . (2009) M.Sc. Thesis in Biochemistry ,College of Sciences, University of Mosul.

30-AL-Taee,M,B,(2003). M.Sc. Thesis in chemistry ,College of Sciences, University of Mosul.Iraq. 